\title{
Space-time epidemiology and effect of meteorological parameters on influenza-like illness in Phitsanulok, a northern province in Thailand
}

\author{
Prakash Madhav Nimbalkar, ${ }^{1}$ Nitin Kumar Tripathi ${ }^{2}$ \\ ${ }^{1}$ Department of Geoinformatics, Cartography and Remote Sensing, University of Warsaw, \\ Warsaw, Poland; ${ }^{2}$ School of Engineering and Technology, Asian Institute of Technology, \\ Klong Luang, Thailand
}

\begin{abstract}
Influenza-like illness (ILI) is an acute respiratory disease that remains a public health concern for its ability to circulate globally affecting any age group and gender causing serious illness with mortality risk. Comprehensive assessment of the spatio-temporal dynamics of ILI is a prerequisite for effective risk assessment and application of control measures. Though meteorological parameters, such as rainfall, average relative humidity and temperature, influence ILI and represent crucial information for control of this disease, the relation between the disease and these variables is not clearly understood in tropical climates. The aim of this study was to analyse the epidemiology of ILI cases using integrated methods (space-time analysis, spatial autocorrelation and other correlation statistics). After 2009s $\mathrm{H}_{1} \mathrm{~N}_{1}$ influenza pandemic, Phitsanulok Province in northern Thailand was
\end{abstract}

Correspondence: Prakash Madhav Nimbalkar, Department of Geoinformatics, Cartography and Remote Sensing, Faculty of Geography and Regional Studies, University of Warsaw, Krakowskie Przedmiescie 30, 00927 Warsaw, Poland.

Tel. +48.225521507 - Fax: +48.225521521 .

E-mail: p.nimbalkar@uw.edu.pl

Key words: Influenza-like illness; Epidemiology; Space-time analysis; Weather variability; Thailand.

Acknowledgments: the authors would like to express their sincere gratitude to Phitsanulok Province Public Health Department for providing high quality of data for this research. We are also extremely grateful to Provincial Public Health Office, Department of Provincial Administration, Thai Meteorological Department, Department of Disease Control, and Ministry of Public Health, Thailand for the eminent data sharing for academic research. Authors are very grateful to anonymous reviewers of Geospatial Health who guided to improve interpretability of the article.

Received for publication: 10 January 2016.

Revision received: 10 June 2016.

Accepted for publication: 15 July 2016.

(C) Copyright P.M. Nimbalkar and N.K. Tripathi, 2016

Licensee PAGEPress, Italy

Geospatial Health 2016; 11:447

doi:10.4081/gh.2016.447

This article is distributed under the terms of the Creative Commons Attribution Noncommercial License (CC BY-NC 4.0) which permits any noncommercial use, distribution, and reproduction in any medium, provided the original author(s) and source are credited. strongly affected by ILI for many years. This study is based on ILI cases in villages in this province from 2005 to 2012 . We used highly precise weekly incidence records covering eight years, which allowed accurate estimation of the ILI outbreak. Comprehensive methodology was developed to analyse the global and local patterns of the spread of the disease. Significant space-time clusters were detected over the study region during eight different periods. ILI cases showed seasonal clustered patterns with a peak in 2010 ( $\mathrm{P}>0.05-9.999$ iterations). Local indicators of spatial association identified hotspots for each year. Statistically, the weather pattern showed a clear influence on ILI cases and it strongly correlated with humidity at a lag of 1 month, while temperature had a weaker correlation.

\section{Introduction}

Influenza-like illness (ILI) is a highly contagious acute respiratory disease capable of triggering outbreaks ranging from local epidemics to global pandemics (Sakai et al., 2004; WHO, 2014). It is responsible for a high contribution of all influenza cases (Kimura et al., 2011; Prachayangprecha et al., 2015), yet it is not well understood and the clinical symptoms are not easily differentiable from other respiratory diseases (Laguna-Torres et al., 2009; Tamerius et al., 2011). ILI has been attributed by wide range of respiratory diseases, predominantly attributed by viruses such as influenzas A and B (Yang et al., 2012; Zhang et al., 2014). The diagnostic approach for ILI is the same as that of influenza (Kelly and Birch, 2004), but all ILIs are not caused by influenza virus. However, more than $70 \%$ of all ILI cases diagnosed during the epidemic of June 2009 to January 2010 were due to the influenza A, subtype $\mathrm{H}_{1} \mathrm{~N}_{1}$ virus (Yang et al., 2012). The clinical symptoms of ILI present as a sudden onset of fever $\left(\geq 38^{\circ} \mathrm{C}\right)$, body ache with one of the typical respiratory symptoms such as sore throat and cough (CDC, 2016). The infection occurs around the year in tropical regions and in the winter to spring period in temperate regions Like common influenza, it circulates around the globe rapidly (Birch and Kelly, 2004; Yang et al., 2012). According to the World Health Organization (WHO) surveillance, ILIs spread from infected to healthy persons through fomites in a close proximity, and the human host is the utmost vector and carrier (Bollaerts et al., 2013; Yang et al., 2015). Therefore, a full understanding of the connection with the vector has an enormous potential with respect to quantification of ILI dissemination and its spatial dynamics, knowledge of which would strongly contribute to control of the disease (Viboud et al., 2006).

This study occupies itself with the general forms of respiratory diseases and spatial dynamic studies, but it deals predominantly with influenza since it includes a larger portion of ILI cases in peak seasons (Yang et al., 2015). However, Lowen and Palese (2009) recognised that 
ILIs are mixed infections consisting of adenoviruses, respiratory syncytial viruses (RSV), enteroviruses, human meta-pneumo viruses and parainfluenza viruses besides classical the influenza viral strains. Recent epidemiological modelling has greatly improved our knowledge of viral evolution and the related complex antigenic evolution, but there is also a need to understand the spatio-temporal aspects of the vectorborne respiratory diseases (Nelson and Holmes, 2007; Soebiyanto et al., 2015). Furthermore, in a series of monitored influenza studies with special reference to ILI viruses, it is stressed that ILI spatio-temporal dynamics must be clarified within the broader aspect of respiratory infections (Bollaerts et al., 2013). In tropical and subtropical settings, ILI epidemics occur more frequently with high temporal variability. However, people are not aware about the burden of morbidity which negatively affects the socio-economic strata involved (Viboud et al., 2006; Simmerman and Uyeki, 2008; Simmerman et al., 2009; Yang et al., 2015). Although the majority of the viruses which collectively make up the ILIs can be preventable by early vaccinations, e.g., against influenzas A and B (WHO, 2013; Prachayangprecha et al., 2015), there is no such thing as an integrated vaccine to be used for general ILI prevention. The Thai infectious disease control and vaccination programme vaccinates against the majority of seasonal influenza viruses according to global standards (Prachayangprecha et al., 2015). However, these vaccines remain effective only for a year and ILI peaks frequently a year later due to the ability of these viruses to rearrange genomes varying from minor mutations to major antigenic shift under the pressure of human immunity. Since the immune system is not used to new viruses (Morse, 1995), large numbers of people are vulnerable in spite of prior infections and vaccinations (Laguna-Torres et al., 2009; Yang et al., 2015).

Past research of respiratory diseases in the current healthcare settings are limited to hospitalised and laboratory confirmed cases (Russell et al., 2008; Kimura et al., 2011), which are highly likely to underestimate the real situation, thereby hiding the broader view of spatial disease dynamics (Anderson et al., 2012). Additionally, most influenza studies are based on the 1918 and 2009 pandemics (Biggerstaff et al., 2014), so there is still insufficient information on ILI dynamics. Tamerius et al. (2011) have reviewed the effect of seasonality, human immunity and virus survival on the spread of respiratory viruses. Anderson et al. (2012) assessed ILI based on occupation, sex, social contacts and stress levels finding that social contact in public (school, workplace, etc.) and contaminated areas (markets, banks, etc.) are sources of infections. Furthermore, stress, job insecurity and physiological distress impact adversely on the general health, which leads to prevalence of ILI.

Research studies have focused on specific respiratory viral strains from confirmed cases, while less attention has been given to the estimation of ILI space-time dynamics. Numerous studies on the relations between meteorological parameters and influenza have been carried out (Charland et al., 2009; Rambaut et al., 2008). Shaman and Kohn (2009) introduced mathematical modelling to estimate seasonal patterns of Influenza from humidity, while Tamerius et al. (2011) and Zhang et al. (2014) cited studies on influenza susceptibility, epidemiology and aetiology and its relationship with virus types, regional immunity, indoor crowding, climate, and socioeconomic factors. The rate of virus transfer depends on the virus load (carrying capacity) and the surrounding weather condition (Dushoff et al., 2004; Charland et al., 2009; Lowen and Palese, 2009). Influenza viruses can remain infectious up to twelve days on inanimate surfaces, and several respiratory pathogens can be active several months depending on the average relative humidity and temperature (Kramer et al., 2006).

This study assumes a high correlation between ILI and influenza cases and compares weather dynamics in relation to both ILI and other forms of influenza since minor changes in the weather parameter can induce a large number of ILI cases (Shaman et al., 2010; Chong et al., 2015). The overall aim was to elucidate the space-time pattern analysis of ILI from the 2005-2012 epidemic at the village level to identify hotspots with a view to support possible rearrangement of existing strategies and improvising the methods. We studied the situation in Phitsanulok, a northern Thai province where high ILI morbidity (250.55 per 100,000 population) was reported by the Provincial Health Department. This put Phitsanulok Province third with respect to this disease in the top ten provinces after Bangkok.

\section{Materials and Methods}

\section{Study area}

Phitsanulok Province, Thailand, comprising 9 districts and 991 villages over an area of $10,815 \mathrm{~km}^{2}$ (Figure 1). The province, situated around latitude $100 \mathrm{E}$ and longitude $17 \mathrm{~N}$, is mostly covered with forests and mountains and it has mixed weather with a daily average relative humidity ranging from 60 to $87 \%$, high rainfall and strong temperature variability. An administrative boundary map was collected from Thai Ministry of the Interior.

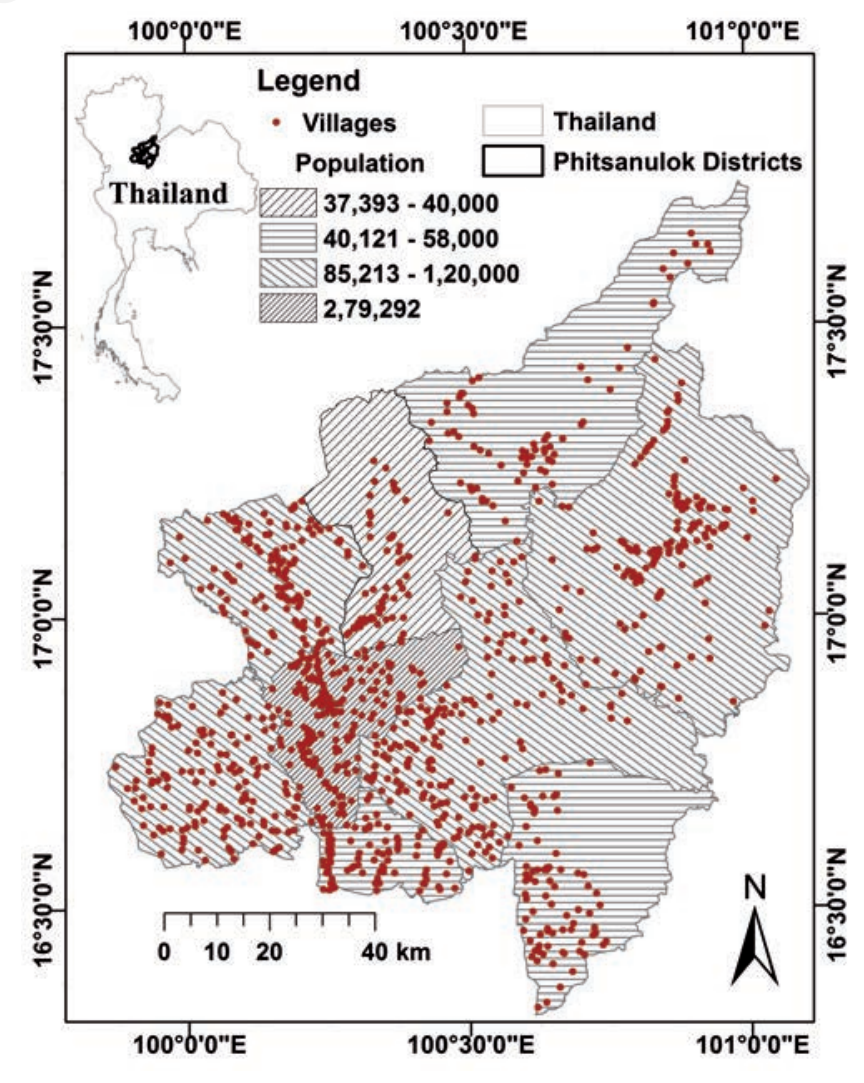

Figure 1. Phitasanulok Province, Thailand, with villages and population distribution. 


\section{Case data}

The Provincial Health Department provided case data for 991villages with ID codes that could be linked with administrative units in a computerised shapefile. Standard case definitions included a sudden onset of fever $\left(\geq 38^{\circ} \mathrm{C}\right)$ together with one respiratory symptom, such as cough, sore throat or body ache. Cases with known cause of respiratory disease (e.g. pneumonia) were excluded. The Thai Ministry of Public Health collects ILI incidence data nationally with high coverage and quality, however this study used only regional, provincial data from 2005-2012 available from regional health centres, municipality hospitals and private clinics responsible for reporting ILI case data to the central provincial health office on a daily basis. This study also focused on the distribution of ILI cases in different age groups and with different occupations. Therefore, details of age and occupation were collected along with case counts.

\section{Meteorological determinants}

Meteorological data (rainfall, humidity and temperature) were collected from Thai Meteorological Department. Data covering eight years (2005-2012) were used to find correlations between cases and weather parameters. IBM SPSS 20 (http://www-01.ibm.com/support/ docview.wss?uid=swg21509012) statistical software was used to analyse the Pearson's correlation coefficient sing a level of significance $<0.05$. The ILI case count for the years 2005 and 2006 was too low to show in the graph, therefore, only the period 2007-2012 was plotted (Figure 2).

\section{Data analysis}

Geographical information systems (GIS) were used to support the investigation of the geospatial analysis. The space-time term refers to the simultaneous detection of spatio-temporal clusters using dedicated SaTScan package tools (Kulldorff, 2005). To detect spatial hotspots in the region, spatial autocorrelation methods in the GeoDa software package (https//geodacenter.asu.edu/software/downloads) was used.

\section{Space-time clusters}

The question whether ILI cases were distributed randomly over space and/or time and whether there was any significant clustering pattern in region was investigated using SaTScan V9.4.2 open source software (http//www.satscan.org/). Space-time statistics explicitly considers the connections between space and time in three-dimensional (3-D) space noting observed verses expected cases inside a circular window. Space-time analysis is based on the use of a cylindrical window, where the base of cylinder represents the geographical location and the height the temporal extent of a cluster. The method is a retrospective analysis using Poisson discrete statistics which considers population under risk, where the null hypothesis (to be rejected) defines cases as distributed randomly over space and time (Kulldorff et al., $1998,2005)$. Information of a village population was used to find the population under risk. The population data for the median year (2009) was selected to avoid the bias of population change during the study period. Using retrospective analysis to find significant cluster(s), the number of collected ILI cases by date and ID-sourced village location were applied using 999 permutations of the Monte Carlo statistics approach. The SaTScan application was run separately for each year. To consider all temporal cluster possibilities, weekly data were selected in annual cycles starting in April since the number of ILI cases was at lowest in that month. To avoid overestimation of cases from any potential cluster origin in region (this also allowed the detection of secondary significant clusters), the maximum spatial cluster size was allowed to grow considering the number of people and geographical size of region, using $50 \%$ of the population at risk as default value.

\section{Spatial autocorrelation}

The open source GeoDa software was set to run for 999 permutations analysing the data by Global Moran's I global and local indicators of spatial association (LISA) (Anselin, 2010). The outcome was considered significant at $\mathrm{P}>0.05$ to reject the null hypothesis. Spatial analysis (SA) is a purely SA that compares degree of correlation between observed cases to their geographical locations considering the population under risk. Moran's $I$ is a global statistics used to find global phenomenon over the whole study area where the null hypothesis states that cases geographical locations are not correlated, i.e. random. Moran's I near 1 indicate strong cluster pattern and values approaching to zero refers to a random pattern. Neighbouring areas have high order of contingency. To incorporate this, neighbourhood weight matrix estimated using the K-nearest neighbours method, which defines the spatial relation between neighbours using distances between them. We estimated the matrix at the threshold distance, i.e. the maximum distance between two neighbouring villages (Anselin, 2005, 2010). In Moran's I; Bayesian smoothing rate was estimated from the information of ILI cases and population of each village; smoothing of the rate was done to avoid bias of the less populated villages with high morbidity. We scanned the entire dataset over the region with Moran's $I$ window and report significant clusters with high-high and low-low values including insignificant locations (random) using the Z score statistic (Anselin, 2005, 2010). We used the top and bottom values of significance (two-tailed test) to achieve a high level of significance (http://www.ats.ucla.edu/ stat/mult_pkg/faq/general/tail_tests.htm).

\section{Hotspot-local spatial autocorrelation}

Hotspot is a local phenomenon provided by local SA, which identifies spatial clustering in a specified area. Although there may be a global clustering of a disease in an entire study region, this disease may

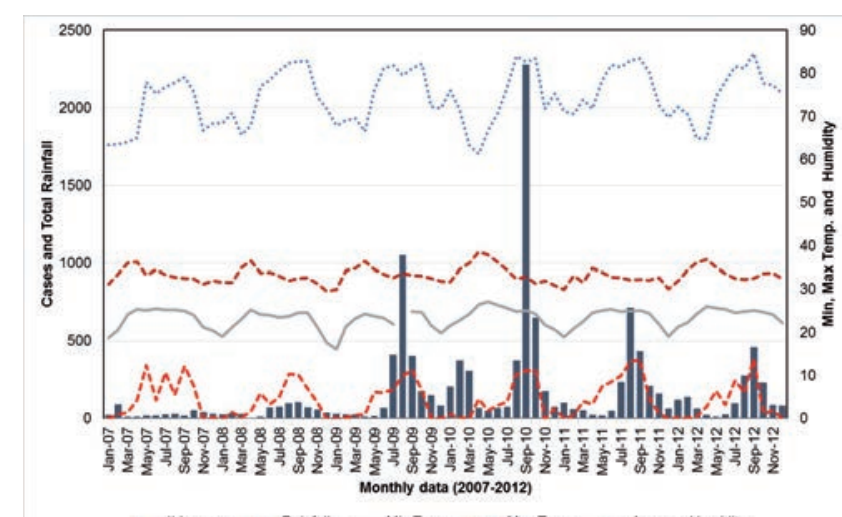

Figure 2. Monthly numbers of influenza like illness (ILI) cases and meteorological data in Phitasanulok Province, Thailand, for the period 2007-2012. Case numbers from the years 2005 and 2006 were excluded due to low count. The average relative humidity peaks were well synchronised with ILI cases-highest peak observed in September 2010. The rainfall peaks were best correlated in 2008 and 2011-2012. 
spread in various ways by local phenomena, e.g., due to favourable/unfavourable geographical or social conditions in the same region. As global Moran's I cannot detect the local clustering, LISA (Anselin, 2010) was utilized to identify local phenomenon of spatial distribution. This approach allows the decomposition of the global Moran's I of spatial association to local individual observation at certain threshold areas. We analysed cases with location information within a $10-\mathrm{km}$ radius considering all possible combinations of disease spread to find hotspots as described by various scientists (Crighton $e t$ al., 2008; Anselin, 2010). LISA was run for each year due to high spatial variation across years with a randomization of 999 permutations. We scanned village locations where the magnitudes of ILI morbidity were particularly high or low to relate with an identical or uniform condition. High-high magnitude clusters defined the locations as ILI hotspots.

\section{Results}

\section{Influenza-like illness incidence}

There were a total 12,360 ILI cases in Phitsanulok Province during the study period. Based on this figure, the analysis gives an average yearly morbidity of 286 cases per 100,000 population for the whole period $2005-2012$. The highest morbidity $(580$ per 100,000$)$ was observed in 2010. Despite the fact that 2009 was the year of the $\mathrm{H}_{1} \mathrm{~N}_{1}$ pandemic, which totalled 2474 ILI cases (fourfold that of the previous year). The number of cases in 2010 increased to 4718 (twofold of that in 2009). Thus, within two years from 2008 to 2010 the number of cases increased eight times. In the whole study period, the number of ILI cases in children less than five years old, school children and students collectively contributed to an average of $78.1 \%$. The ILI distribution among different age groups revealed that children and students were highly vulnerable to ILI infections (Figure 3 ), presumably due to different immune status and different social activity in children and adults. Unexpectedly, the percentage of ILI in children less than five decreased in 2009 and 2010 even if the prevalence increased in 2011 and 2012. The percentage of students with ILI increased abruptly in 2009 (Figure 3). Disease distribution between occupations, such as public servants, manual labour, farmer and people out of work did not reveal any particular pattern.

\section{Space-time clustering}

In SaTScan, the Poisson retrospective model detected clustering by comparing observed versus expected clusters. Spatial and temporal patterns of ILI were analysed simultaneously from April to April across the whole study period to detect significant seasonal clustering cycles. Unlike influenza, other respiratory viruses generally circulate in the region for 10-15 days. To avoid false clustering results, the duration of aggregation was kept from 10 days to a possible maximum extent. We recorded eight significant spatio-temporal cluster periods in the entire 8-year study period in this study region. The date of initial signs of clustering varied across the region from year to year, but the majority of clusters were detected in the June-July period (Figure 4). SaTScan inspects millions of potential clusters (primary and secondary), from which only significant clusters (primary) with spacetime dimensions were reported from the village, based on their IDs. In the temperate region of Phitsanulok, primary clusters at significant confidence levels $(\mathrm{P}=0.001$ to $<0.05)$ were localised (Table 1$)$. The number of ILI cases recorded in the clusters was diverse year to year also with temporal durations ranging from 1 to 10 months. In the whole study period an average of 24.3 -km radius of disease outbreaks (clusters) were observed. We were able to detect the eight space time cluster in region; only one cluster from 2007 showed the high temporality of 10 month, while the majority of clusters showed 3 -month temporal duration.

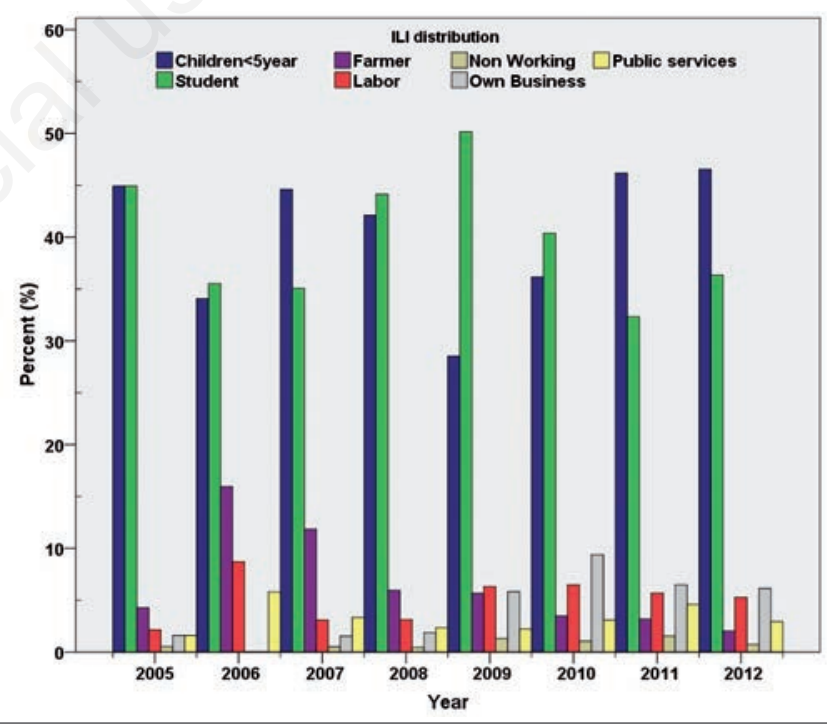

Figure 3. The number of influenza like illness (ILI) cases in Phitasanulok Province, Thailand, classified by occupation.

Table 1. Significance of identified influenza-like illness space-time clusters in Phitsanulok Province, Thailand.

\begin{tabular}{|c|c|c|c|c|c|}
\hline Village name (ID) & Start date & End date & Radius (km) & $\mathbf{P}$ & Cases (n) \\
\hline Phai Khom Rattanaram (65011001) & July 05, 2005 & December 01, 2005 & 24.97 & 0.001 & 114 \\
\hline Leam doo (65080307) & January 31, 2007 & March 01, 2007 & 16.88 & 0.001 & 49 \\
\hline Ngiw Ngam (65012004) & June 06, 2007 & March 31, 2008 & 28.68 & 0.001 & 257 \\
\hline Phai kho don (65011902) & June 05,2008 & January 30, 2009 & 16.85 & 0.001 & 323 \\
\hline Bueng Phrao (65081003) & July 05, 2009 & October 02, 2009 & 27.41 & 0.001 & 1184 \\
\hline Tha pho (65011208) & September 03, 2010 & October 02,2010 & 24.97 & 0.001 & 1653 \\
\hline Pongsathon-Chin laap (65010707) & July 11, 2011 & October 08, 2011 & 24.46 & 0.001 & 1067 \\
\hline Krap Phuang Tai (65060104) & August 04, 2012 & November 01, 2012 & 30.03 & 0.001 & 543 \\
\hline
\end{tabular}




\section{Spatial autocorrelation-Moran's I}

GeoDa detected spatial clusters in all years of the study except in 2009 , the strength of clusters indicated by Z-values and Moran's $I$. The higher the Z-value, the higher the strength and intensity of the cluster investigated. The observed $\mathrm{Z}$ value in 2010 was 48.28 , which was the highest cluster pattern during the whole period. High values of Moran's $I$ were observed in 2007 and 2010, while the lowest index was seen in 2009 , indicating a random pattern at that year.

\section{Hotspot detection-local indicators of spatial association}

The LISA tool detected geographically homogenous high-high and low-low values in the study area, the localisation of which is shown in Figure 5 . These spatially significant local clusters were missed by global Moran's I statistic. However, some of the local clusters were of unexpected high strength (z-value) and they also correlated with the global Moran's I (Table 2). This index was at its lowest levels of significance in 2006 and 2009, but appeared as equivalent disperse cluster patterns on the LISA maps by visual inspection. However, the Z-value gives only information about the cluster strength and the LISA cluster maps differ at scale and geographical extent. For instance, the 2006 cluster did not show a strong aggregation of village points, and there were many highhigh and low-low clusters in 2009. The year 2010 showed the highest, well-defined cluster pattern across the study period, while 2011 and 2012 showed increased spread with low-low clusters in the southern region of Phitsanulok Province.

\section{Weather variability}

Pearson's correlation statistics was used to analyse, on a weekly basis, the relation between ILI cases and rainfall, minimum temperature, mean temperature, maximum temperature, change in temperature and average relative humidity. We assumed that a change in weather parameters take some duration to create favourable environmental condition (local level) for the infection to start spreading. Best correlation coefficient was observed with average humidity, in particular after 1-month lag (Table 3 ) at $\mathrm{P}$ values varying between 0.05 and 0.01 . These lag cases ( $t-1)$ were highly correlated with the average humidity. Furthermore, high negative correlation was observed with regard to change in temperature. However, while maximum temperature affected the $\mathrm{t}-1$ cases negatively, we did not find any significant relation with mean temperature. Rainfall correlated strongly without time shift (t), indeed stronger than $\mathrm{t}-1$. Exceptionally, the cases recorded in 2006 were not correlated with rainfall. Minimum temperature in 2009 revealed higher negative correlation $(-0.80)$ with cases at $t$, which was significant to note; also correlation with cases at t from the whole study period showed negative (-.18) correlation with average rainfall (Table 3).

The coefficients showed a positive correlation between the weekly number of cases on the one hand and average humidity and average rainfall on the other. Furthermore, to highlight the relation between peak values of humidity and rainfall with peaks of the weekly number of cases, they were plotted together (Figure 6). When pivot table data filtered for the top 10 values of humidity for the entire study period were used, only the number of cases exceeding 50 per week were



Figure 4. Detected influenza like illness space-time clusters in Phitasanulok Province, Thailand, including their start dates.

Table 2. Results of global Moran's $I$ and local indicators of spatial association measurements.

\begin{tabular}{|c|c|c|c|c|c|}
\hline Year & Villages (n) & P & Moran's I & $\mathbf{Z}$ & Distribution pattern \\
\hline 2005 & 115 & 0.001 & 0.598 & 36.61 & Cluster \\
\hline 2006 & 84 & 0.001 & 0.661 & 20.58 & Cluster \\
\hline 2007 & 204 & 0.002 & 0.632 & 41.29 & Cluster \\
\hline 2008 & 289 & 0.002 & 0.559 & 43.11 & Cluster \\
\hline 2009 & 664 & 0.001 & 0.039 & 12.68 & Random \\
\hline 2010 & 831 & 0.001 & 0.696 & 48.28 & Cluster \\
\hline 2011 & 613 & 0.004 & 0.583 & 39.21 & Cluster \\
\hline 2012 & 603 & 0.005 & 0.432 & 37.68 & Cluster \\
\hline
\end{tabular}


selected for plotting. These 10 top average humidity accounted for 5212 ILI cases (up to $42.2 \%$ of the cases). In the graph shown in Figure 6, rainfall had similar spikes as the number of ILI cases, predominantly in the weeks of August and September in 2009 and 2010. However, in the initial years of study (2005-2008), no major spikes were observed. In 2010, seven ILI spikes were observed which amounted to $35 \%$ of all spikes, whereas in 2009 and 2011 five spikes were observed each year.

\section{Discussion}

This study integrated multiple tools to analyse ILI space-time epidemiology in Phitsanulok. Using various spatiotemporal analysis methods and correlation statistics, such as Moran's I and LISA, with meteorological parameters effectively mapped and determined the spatial epidemiology of ILI during 2005-2012 in local tropical settings.

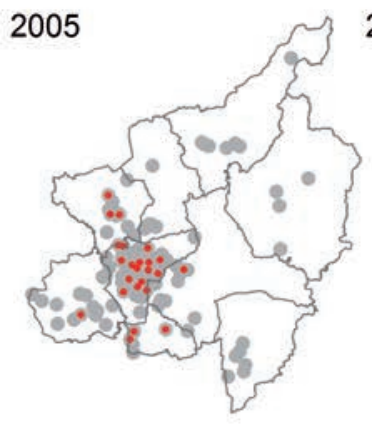

2006



2009

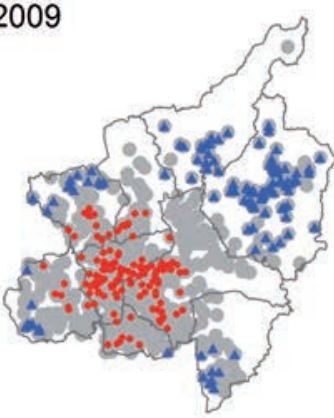

2010

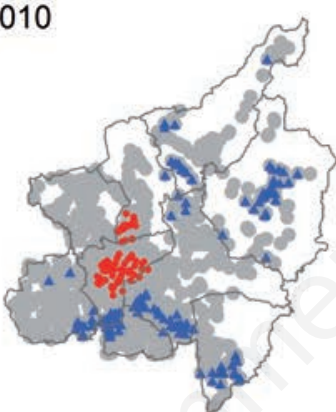

Legend

ILI Cluster • High-high $\wedge$ Low-low $\square$ Districts of Phitanulok - Insignificant



2011

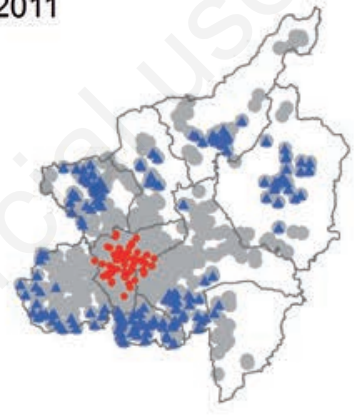

2007

2008

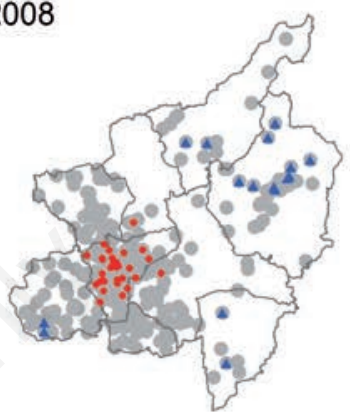

2012

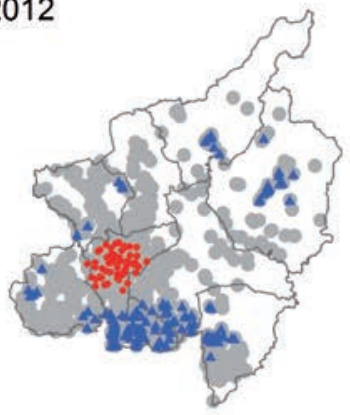

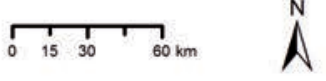

Figure 5. Influenza like illness (ILI) clusters (hotspots) in Phitasanulok Province, Thailand, for the period 2005-2012.

Table 3. Results of evaluation using Pearson's correlation coefficients.

\begin{tabular}{|c|c|c|c|c|c|c|}
\hline Year & Lag time & $\begin{array}{l}\text { Average } \\
\text { rainfall }\end{array}$ & $\begin{array}{l}\text { Minimum } \\
\text { temperature }\end{array}$ & $\begin{array}{l}\text { Maximum } \\
\text { temperature }\end{array}$ & $\begin{array}{l}\text { Average } \\
\text { humidity }\end{array}$ & $\begin{array}{l}\text { Change in } \\
\text { temperature }\end{array}$ \\
\hline 2005 & One month $(\mathrm{t}-1)$ & 0.196 & 0.185 & -0.124 & $.425^{* *}$ & $-.399 * *$ \\
\hline 2006 & & 0.133 & 0.124 & $-0.303^{*}$ & $.412^{* *}$ & $-.419 * *$ \\
\hline 2007 & & -0.036 & $-0.304^{*}$ & $-0.395^{* *}$ & 0.021 & 0.024 \\
\hline 2008 & & $0.519 * *$ & $0.284^{*}$ & -0.173 & $.672^{* *}$ & $-.599 * *$ \\
\hline 2009 & & $0.276^{*}$ & $-0.327^{*}$ & -0.156 & $.533^{* *}$ & $.295^{*}$ \\
\hline 2010 & & $0.438^{* *}$ & 0.041 & $-0.273^{*}$ & $.504^{* *}$ & $-.441^{* *}$ \\
\hline 2011 & & $0.480^{* *}$ & $0.363^{* *}$ & 0.002 & $.556 * *$ & $-.459 * *$ \\
\hline 2012 & & $0.469 * *$ & 0.021 & $-0.563^{* *}$ & $.571^{* *}$ & $-.574 * *$ \\
\hline 2005-2012 & & $0.229 * *$ & -0.058 & $-0.112^{*}$ & $.361^{* *}$ & -0.006 \\
\hline 2005 & No lag (t) & $0.333^{*}$ & 0.1 & $-0.272^{*}$ & $.473^{* *}$ & $-.465^{* *}$ \\
\hline 2006 & & 0.08 & $0.295^{*}$ & -0.01 & $.378^{* *}$ & $-.452^{* *}$ \\
\hline 2007 & & $0-.248 *$ & -0.184 & -0.184 & -0.232 & $.280 *$ \\
\hline 2008 & & $0.435^{* *}$ & 0.148 & $-0.315^{*}$ & $.593^{* *}$ & $-.564 * *$ \\
\hline 2009 & & $0.426^{* *}$ & $-0.805^{* *}$ & -0.048 & $.455^{* *}$ & $.821^{* *}$ \\
\hline 2010 & & $0.404^{* *}$ & 0.067 & -0.232 & $.412^{* *}$ & $-.414^{* *}$ \\
\hline 2011 & & $0.632^{* *}$ & $0.295^{*}$ & -0.057 & $.545^{* *}$ & $-.426^{* *}$ \\
\hline 2012 & & $0.507^{* *}$ & -0.011 & $-0.417^{* *}$ & $.519 * *$ & $-.400^{* *}$ \\
\hline 2005-2012 & & $0.249 * *$ & $-0.181^{* *}$ & -0.08 & $.313^{* *}$ & $.149^{* *}$ \\
\hline
\end{tabular}


Changes of meteorological parameters are highly frequent, complex and abrupt in tropical settings. Even small changes in the weather characteristics can trigger pathological changes in large numbers of peoples vulnerable to respiratory diseases (Tamerius et al., 2011). Therefore, we aimed to find the relationship between weather variability and the number of ILI cases. In this endeavour, we had great support GIS, which contributed by providing user-friendly geospatial analytic techniques suitable to carry out research on disease dynamics more efficiently than previously. Many spatial statistical standard tools are now available in GIS packages, which lend themselves well to spatial and temporal analysis (Anselin, 2010).

The key findings of this study are that children under the age of five and students (5-14 years old) are the most susceptible age groups (Figure 3), which is supported by Simmerman et al. (2009) and Anderson et al. (2012), who found that children are most susceptible to ILI infections. Although routine child vaccination and selective seasonal influenza vaccinations programmes cover major respiratory diseases in the above mentioned age groups, we found that ILI cases represented $72.2 \%$ of the cases in the study, while children under five and students below 14 account for only $23 \%$ of the study population. i.e. $300 \%$ higher risk than other age groups. There are no vaccines available for respiratory viruses, additionally human RSV, rhinovirus and parainfluenza viruses, which contribute to ILI due to rapid change in the genome which cause repeated infections in the same population (Yang et al., 2012). Despite advanced precautionary measures, ILI imposes serious concern with respect to young adults, possibly due to lower immunity, high social communication and low precautionary measures in school children (Anderson et al., 2012). Farmers, general labourers, small-scale private business people, public servants and the unemployed contribute collectively to $18-22 \%$ of all ILI cases (Figure 3). These classes showed less susceptibility to ILI than students (Anderson et al., 2012). Moreover, awareness at work, vigilance and precautionary working environment have shown that it is possible to interrupt the spread of the disease.
Though spatial epidemiological studies on ILI are less explored due to lack of laboratory confirmation, they offer a broader view of the pattern of respiratory diseases. Therefore, empirical comparison between contributing respiratory viruses to ILI and collected cases of ILIs would be useful. As influenza virus generally occupies the major part of ILI cases, we compared some influenza studies. Overall, influenza made up $30 \%$ of the ILI cases, but could, in peak month, reach above $70 \%$ (Kimura et al., 2011; Yang et al., 2012). In general, elevated vaccination and preventive measures after the 2009 pandemic, the number of cases fell in the tropical regions in 2010 (Yang et al., 2012), whereas we observed the highest increase of cases in 2010 (Figure 2). This could be due to increased viral survival due to weather conditions, a higher virulence factor in the region or the fact that immunity developed through prior infections is temporary (Dushoff et al., 2004). Male and female percentage of ILI cases in Thailand were different for each year investigated, collectively $51.2 \%$ for males and $48.8 \%$ for females over the whole 8-year period, even though the female population was higher (97 males to 100 females). However, this difference is not statistically significant.

Numerous mechanisms have been proposed to find spatial and/or temporal patterns of respiratory diseases, but simultaneous focus on space-time patterns are lacking, especially for ILI. Our work shows that the integrated assessment of tools can be used to effectively map the dynamics of ILI in 3-D space, global space and also for finding the hotspots. Eight space-time clusters were identified at $\mathrm{P}<0.05$. Major clusters were shown to build in the rainy season from June to August, continuing for 3 months. In contrast, clusters that started in the month of January or March persisted only for a month (Figure 3). In 2009, smaller peaks started in July with a maximum in September, the same pattern was observed in the period 2010-12 (Figure 2). Space-time retrospective scanning window revealed that ILI has the potential capability to disseminate through a $16-30 \mathrm{~km}$ radius area, which approximately covers up to $50 \%$ of the study area, indicating that people in the study area must have a high social interconnectivity. The spatio-temporal

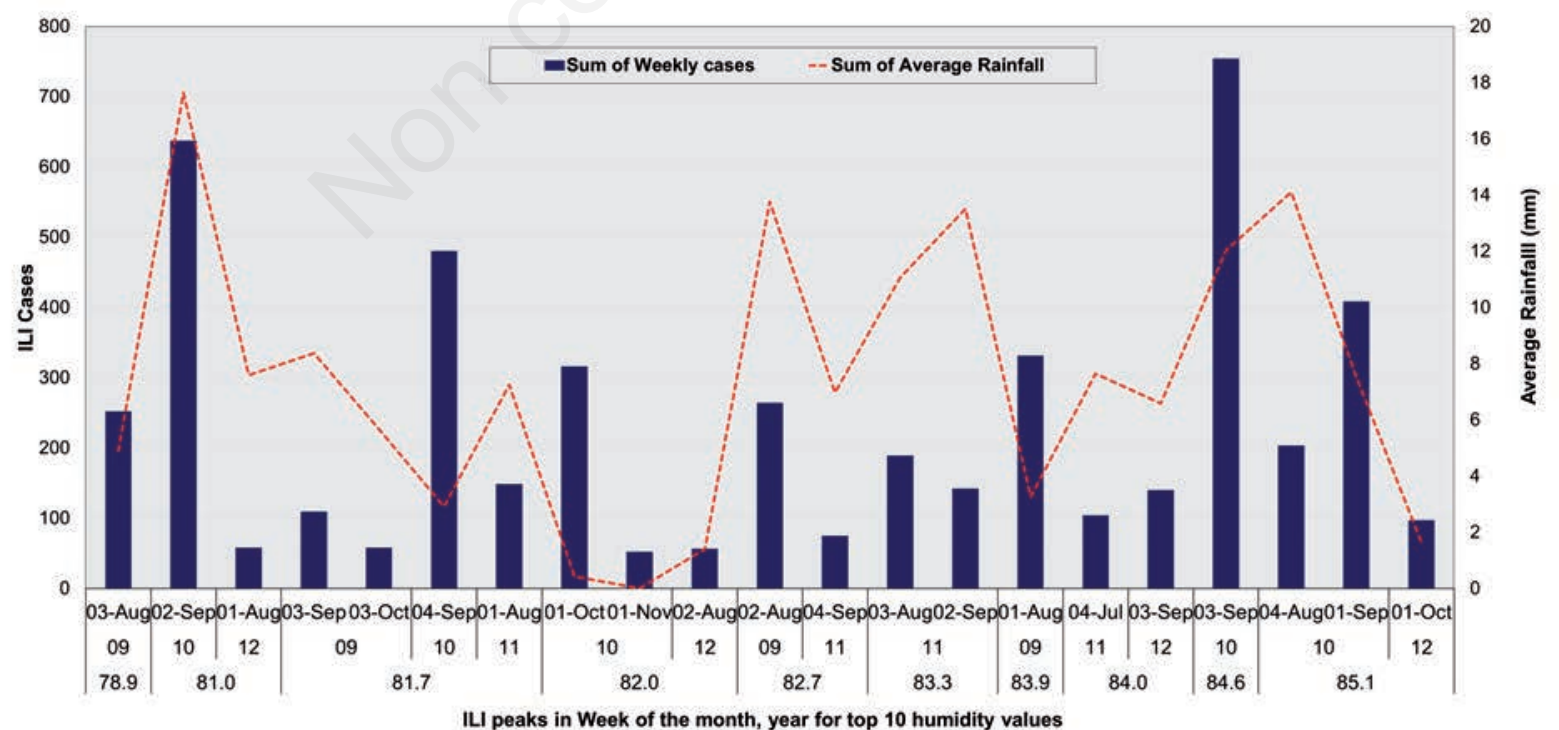

Figure 6. Weekly spikes of the number of influenza like illness (ILI) cases in Phitasanulok Province, Thailand, for the top 10 humidity values. Labels on horizontal $(x)$ axis: first row is the number of week in that month, second row represents the year (yy), and third row stands for average humidity (\%). The figure denotes the top 10 peaks of humidity where ILI cases have more than $\mathbf{5 0}$ patients; weekly average rainfall $(\mathrm{mm})$ is plotted as well. 
clustering in 2007 and 2008 persisted for 8 and 10 months, respectively, eventually spreading over an area of 28.7 to $16.8 \mathrm{~km}$ radius. However, the rate of viral propagation was actually low: 257 to 323 cases, respectively (Table 1). The clusters starting in the last week of January 2007 and June 2008 clearly coincide with influenza A spikes as in the study by Simmerman et al. (2009), who included only influenza A cases confirmed in the laboratory. As influenza A is the most common pathogen of the seasonal circulating viruses (Simmerman et al., 2009), the majority of these infections could have emerged from this virus rather than from influenza B. However, there is not sufficient data to draw clear lines regarding the dynamics between these two virus types.

In a purely spatial approach, Moran's I could elucidate the evolving pattern of ILI in the region. We recognised that there was distinctive pattern in spread of ILI virus across the region for all the years identified using spatial statistics. The year 2009 showed a random pattern, possibly due to ILI closely following the $\mathrm{H}_{1} \mathrm{~N}_{1}$ pandemic, leaving behind most of the districts and remote villages with ILI. The longest temporal window (8 months) appeared in 2007. It had a high z-score indicating a strong aggregation pattern (Table 2). The 2010 space-time cluster included up to 1,653 cases within the span of one month. All the years studied, except 2009, showed clustered patterns. The application of LISA is known for its ability to effectively locate the spatially active local clusters (Figure 5), which are missed (by default) by the global Moran I (Jeefoo et al., 2011). Using the LISA approach, we observed two highhigh clusters marking a hotspot in 2010 . This was the highest density in our material, but we observed in the period of 2010-2012 also a compact, low-low cluster pattern with a $\mathrm{P}=0.001$. The visual analysis of this pattern showed clusters with highly defined boundaries (Figure 5). Although the years 2005-2007 showed high-high cluster hotspots, the strength according to visual interpretation noted only a weak aggregation of involved villages, especially in 2006. This finding associates well with the absence of clusters in 2006 according to our space-time analysis. Knowledge of these clusters hold vital information for planning of disease control and vaccination programmes, while yearly cluster maps showed that the pattern was diverse for high-high and low-low clusters with reference to year, geographic extent and number of villages infected. However, Mueang Phitsanulok, the capital of Phitsanulok Province, was affected every year (Figure 5). The high population in the capital (Figure 1), plus yearly increasing migration resulting in overcrowding, produces naturally a high vector mobility. Our findings also suggest that population density is the major factor in disease spread as shown by Charland et al. (2009).

The strength and extent of ILI epidemics are influenced by factors such as virulence, relative immunity of the population, crowding and the weather conditions (Prachayangprecha et al., 2015). Pearson's correlation coefficient between meteorological parameters and number of ILI cases showed different significance levels (Table 3). We are in agreement that higher humidity has positive relation (directly proportional) to the number of ILI cases (Soebiyanto et al., 2015; Tang et al., 2010). Additionally, humidity had a highly significant correlation with the number of ILI 1 month later, possibly due to increasing humidity decreases immunity to the disease and thus increasing virus survival (Tamerius et al., 2013). We also found high humid-rainy conditions coinciding with ILI peaks as found by these authors. To explore more about the humidity relation, we filtered the 10 high values of average humidity. Peaks of ILI cases coincided with high humidity values and average rainfall in the same period. For the top 10 humidity peaks, ILIs accounts up to $40 \%$ of cases (Figure 6). This study shows that rainfall has an immediate impact on ILI cases, possibly due to following the high humid conditions. Although rainfall has shown a relatively low correlation coefficient (Tamerius et al., 2013), some studies suggest that rainfall and higher humidity increase indoor crowding leading to an aggregation of the human population as well as favouring virus propagation (Lofgren et al., 2007). In agreement with Prachayangprecha et al. (2015), we observed that major ILI peaks occurred in the rainy and early winter season trailing from July and sustaining high rates up to December causing a major ILI spike, in some years two (Figure 6). Another small ILI spike was observed in the February-March period, while it appeared as a flat line in April, a drop in the number of cases also found by Kimura et al. (2011).

As often observed in temperate regions (Viboud et al., 2006; Soebiyanto et al., 2015), respiratory viruses have an inverse association with lower temperatures. Besides higher humidity, lower temperatures may also facilitate survival and transmission of respiratory pathogens as showed by Lowen et al. (2007). Various factors could facilitate the spread of ILIs, but it clear that rainfall, humidity and low temperatures contribute significantly. Lowen and Palese (2009) report that an average relative humidity level above $80 \%$ block the spread of respiratory virus. However, in contrast, this study observed 18 spikes between 81.0 to $85.1 \%$ of humidity, which accounts for nearly $40 \%$ of all ILI cases in the entire study period. The temporal graph (Figure 2) illustrates that the major ILI spikes were observed in the AugustDecember and the February-March periods.

Increased surveillance activities after the $2009 \mathrm{H}_{1} \mathrm{~N}_{1}$ pandemic in the region, does not relate with rise of ILI cases in 2010, as identified clusters reveal significant viral outbursts and absence of randomness. Moreover, space-time statistical scans and LISA scans (hotspots) in 2007 and 2010 clearly show independent strong linkages between ILI cases and the villages where they live. Space-time clusters and visual interpretation (Figure 2) point out that the July-September period is most common for disease outbreak in the region. In addition, the ILI spike increased substantially in the year following the epidemic, i.e. February-March 2010. ILI has shown a seasonal pattern in the spatiotemporal and correlation analysis. However, one limitation was that small study areas has only one meteorological data centre to test extensive weather variability, so provincial border analysis could be more elaborate if neighbouring province information were provided. In addition, better vaccination data could illustrate broader spatial dynamics and so would future studies planned to integrate socioeconomic factors, such as housing patterns, water sources, economic status, stress level and human migration.

\section{Conclusions}

Humidity, rainfall and temperature play a stimulatory role for ILI in the tropics. An average relative humidity and rainfall have a positive correlation with the number of cases, and so has humidity with 1month lag. Temperature parameters have weak negative correlation with cases numbers. Young (children and older school children) are high-risk age groups, indicating persistent ILI burdens by jointly contributing to a majority of ILI cases, sometimes reaching near $80 \%$ collectively. Spatially, ILI infection clusters have an average radius of 24 $\mathrm{km}$, while most cases are found in the period July-October. The year 2010 was the most significant in the study period since it contributed high cluster strengths, space time clusters and multiple hotspots, even though 2009 was the year of the $\mathrm{H}_{1} \mathrm{~N}_{1}$ pandemic. 


\section{References}

Anderson NJ, Bonauto DK, Fan ZJ, Spector JT, 2012. Distribution of influenza-like illness (ILI) by occupation in Washington State, September 2009-August 2010. PloS ONE 7:e48806.

Anselin L, 2005. Spatial statistical modeling in a GIS environment. In: Maguire D, Goodchild M, Batty M, eds. GIS, spatial analysis and modeling. ESRI Press, Redlands, CA, USA, pp. 93-111.

Anselin L, 2010. Local indicators of spatial association-LISA. Geogr Anal 27:93-115.

Biggerstaff M, Cauchemez S, Reed C, Gambhir M, Finelli L, 2014. Estimates of the reproduction number for seasonal, pandemic, and zoonotic influenza: a systematic review of the literature. BMC Infect Dis 14:480.

Birch C, Kelly H, 2004. The causes and diagnosis of influenza-like illness. Aust Fam Physician 33:305-9.

Bollaerts K, Antoine J, Van Casteren V, Ducoffre G, HENS N, Quoilin S, 2013. Contribution of respiratory pathogens to influenza-like illness consultations. Epidemiol Infect 141:2196-204.

CDC, 2016. Overview of influenza surveillance in the United States. Department of Health and Human Services, Centers for Disease Control and Prevention, Atlanta, GA, USA. Available from: http:/www.cdc.gov/flu/pdf/weekly/overview.pdf

Charland KML, Buckeridge DL, Sturtevant JL, Melton F, Reis BY, Mandl KD, Brownstein JS, 2009. Effect of environmental factors on the spatiotemporal patterns of influenza spread. Epidemiol Infect 137:1377-87.

Chong K, Goggins W, Zee B, Wang M, 2015. Identifying meteorological drivers for the seasonal variations of influenza infections in a subtropical city - Hong Kong. Int J Environ Res Publ Health 12:1560-76.

Crighton EJ, Elliott SJ, Kanaroglou P, Moineddin R, Upshur REG, 2008. Spatio-temporal analysis of pneumonia and influenza hospitalizations in Ontario, Canada. Geospat Health 2:191-202.

Dushoff J, Plotkin JB, Levin SA, Earn DJD, 2004. Dynamical resonance can account for seasonality of influenza epidemics. P Natl Acad Sci USA 101:16915-6.

Jeefoo P, Tripathi NK, Souris M, 2011. Spatio-temporal diffusion pattern and hotspot detection of dengue in Chachoengsao province, Thailand. Int J Environl Res Public Health 8:51-74.

Kimura Y, Saito R, Tsujimoto Y, Ono Y, Nakaya T, Shobugawa Y, Suzuki H, 2011. Geodemographics profiling of influenza $A$ and $B$ virus infections in community neighborhoods in Japan. BMC Infect Dis 11:36.

Kramer A, Schwebke I, Kampf G, 2006. How long do nosocomial pathogens persist on inanimate surfaces? A systematic review. BMC Infect Dis $6: 130$.

Kulldorff M, Athas WF, Feuer EJ, Miller BA, Key CR, 1998. Evaluating cluster alarms: a space-time scan statistic and brain cancer in Los Alamos, New Mexico. Am J Public Health 88:1377-80.

Kulldorff M, Heffernan R, Hartman J, Assunção R, Mostashari F, 2005. A space-time permutation scan statistic for disease outbreak detection. PLoS Med 2:216-24.

Laguna-Torres VA, Gómez J, Ocaña V, Aguilar P, Saldarriaga T, Chavez E, Kochel T, 2009. Influenza-like illness sentinel surveillance in Peru. PloS ONE 4:e6118.

Lofgren E, Fefferman NH, Naumov YN, Gorski J, Naumova EN, 2007. Influenza seasonality: underlying causes and modeling theories. J Virol 81:5429-36.

Lowen AC, Mubareka S, Steel J, Palese P, 2007. Influenza virus transmission is dependent on relative humidity and temperature. PLoS Pathog 3:1470-6.

Lowen A, Palese P, 2009. Transmission of influenza virus in temperate zones is predominantly by aerosol, in the tropics by contact: a hypothesis. PLoS Curr 1:RRN1002.

Morse SS, 1995. Factors in the emergence of infectious diseases. Emerg Infect Dis 1:7.

Nelson MI, Holmes EC, 2007. The evolution of epidemic influenza. Genetics 8:196-205.

Prachayangprecha S, Vichaiwattana P, Korkong S, Felber JA, Poovorawan Y, 2015. Influenza activity in Thailand and occurrence in different climates. Springerplus 4:356.

Rambaut A, Pybus OG, Nelson MI, Viboud C, Taubenberger JK, Holmes EC, 2008. The genomic and epidemiological dynamics of human influenza A virus. Nature 453:615-9.

Russell CA, Jones TC, Barr IG, Cox NJ, Garten RJ, Gregory V, Smith DJ 2008. The global circulation of seasonal influenza A (H3N2) Viruses. Science 320:340-6.

Sakai T, Suzuki H, Sasaki A, Saito R, Tanabe N, Taniguchi K, 2004. Geographic and temporal trends in influenzalike illness, Japan, 19921999. Emerg Infect Dis 10:1822-6.

Shaman J, Kohn M, 2009. Absolute humidity modulates influenza survival, transmission, and seasonality. P Natl Acad Sci USA 106:3243-8.

Shaman J, Pitzer VE, Viboud C, Grenfell BT, Lipsitch M, 2010. Absolute humidity and the seasonal onset of influenza in the continental United States. PLoS Biol 8:e1000316.

Simmerman JM, Chittaganpitch M, Levy J, Chantra S, Maloney S, Uyeki T, Areerat P, Thamthitiwat S, Olsen SJ, Fry A, Ungchusak K, Baggett H, Chunsuttiwat S, 2009. Incidence, seasonality and mortality associated with influenza pneumonia in Thailand: 2005-2008. PloS 0NE 4:e7776.

Simmerman JM, Uyeki TM, 2008. The burden of influenza in East and South-East Asia: a review of the English language literature. Influenza Other Respir Viruses 2:81-92.

Soebiyanto RP, Clara WA, Jara J, Balmaseda A, Lara J, Moya ML, Kiang RK, 2015. Associations between seasonal influenza and meteorological parameters in Costa Rica, Honduras and Nicaragua. Geospat Health 10:144-51.

Tamerius JD, Shama, J, Alonso WJ, Alonso WJ, Bloom-Feshbach K, Uejio CK, Viboud C, 2013. Environmental predictors of seasonal influenza epidemics across temperate and tropical climates. PLoS Pathog 9:e1003194.

Tamerius JD, Nelson MI, Zhou SZ, Viboud C, Miller MA, Alonso WJ, 2011. Global influenza seasonality: reconciling patterns across temperate and tropical regions. Environ Health Persp 119:439-45.

Viboud C, Bjørnstad ON, Smith DL, Simonsen L, Miller MA, Grenfell BT, 2006. Synchrony, waves, and spatial hierarchies in the spread of influenza. Science 312:447-51.

WHO, 2013. Influenza (seasonal). World Health Organization, Geneva, Switzerland. Available from: http:/www.who.int/mediacentre/factsheets/fs211/en/

WHO, 2014. Influenza virus infections in humans. World Health Organization, Geneva, Switzerland. Available from: http://www. who.int/influenza/human_animal_interface/virology_laboratories_an d_vaccines/influenza_virus_infections_humans_feb14.pdf?ua=1

Yang W, Lipsitch M, Shaman J, 2015. Inference of seasonal and pandemic influenza transmission dynamics. P Natl Acad Sci 112:201415012.

Yang X, Yao Y, Chen M, Yang X, Xie Y, Liu Y, Wei L, 2012. Etiology and clinical characteristics of influenza-like illness (ILI) in outpatients in Beijing, June 2010 to May 2011. PloS ONE 7:e28786.

Zhang JC, Liu WD, Liang Q, Hu JL, Norris J, Wu Y, Peng ZH, 2014. Spatial distribution and risk factors of influenza in Jiangsu province, China, based on geographical information system. Geospat Health 8:429-35. 